\title{
Spheromak Impedance and Current Amplification
}

\author{
T.K. Fowler, D.D. Hua and B.W. Stallard
}

\section{January 31, 2002}

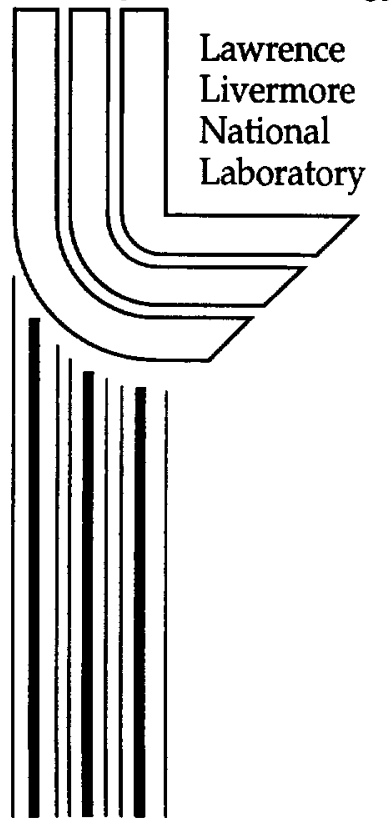




\section{DISCLAIMER}

This document was prepared as an account of work sponsored by an agency of the United States Government. Neither the United States Government nor the University of California nor any of their employees, makes any warranty, express or implied, or assumes any legal liability or responsibility for the accuracy, completeness, or usefulness of any information, apparatus, product, or process disclosed, or represents that its use would not infringe privately owned rights. Reference herein to any specific commercial product, process, or service by trade name, trademark, manufacturer, or otherwise, does not necessarily constitute or imply its endorsement, recommendation, or favoring by the United States Government or the University of California. The views and opinions of authors expressed herein do not necessarily state or reflect those of the United States Government or the University of California, and shall not be used for advertising or product endorsement purposes.

This work was performed under the auspices of the U.S. Department of Energy by the University of California, Lawrence Livermore National Laboratory under Contract No. W-7405-Eng-48.

This report has been reproduced directly from the best available copy.

Available electronically at http://www.doc.gov/bridge

Available for a processing fee to U.S. Department of Energy

And its contractors in paper from

U.S. Department of Energy

Office of Scientific and Technical Information

P.O. Box 62

Oak Ridge, TN 37831-0062

Telephone: (865) 576-8401

Facsimile: (865) 576-5728

E-mail: reports@adonis.osti.gov

Available for the sale to the public from

U.S. Department of Commerce

National Technical Information Service 5285 Port Royal Road

Springfield, VA 22161

Telephone: (800) 553-6847

Facsimile: (703) 605-6900

E-mail: orders@ntis.fedworld.gov

Online ordering: http://www.ntis.gov/ordering.htm

OR

Lawrence Livermore National Laboratory

Technical Information Department's Digital Library

http://www.llnl.gov/tid/Library.html 
Spheromak Impedance and Current Amplification

T. K. Fowler, D. D. Hua and B. W. Stallard

January 31, 2002 Revised

\begin{abstract}
It is shown that high current amplification can be achieved only by injecting helicity on the timescale for reconnection, $\tau_{\mathrm{REC}}$, which determines the effective impedance of the spheromak. An approximate equation for current amplification is:$$
\mathrm{dI}_{\mathrm{TOR}}{ }^{2} / \mathrm{dt} \quad \approx \quad \mathrm{I}^{2} / \tau_{\mathrm{REC}} \quad-\quad \mathrm{I}_{\mathrm{TOR}}{ }^{2} / \tau_{\mathrm{CLOSED}}
$$

where $I$ is the gun current, $I_{\text {TOR }}$ is the spheromak toroidal current and $\tau_{\text {CLOSED }}$ is the ohmic decay time of the spheromak. Achieving high current amplification, $\mathrm{I}_{\mathrm{TOR}}>\mathrm{I}$, requires $\tau_{\mathrm{REC}}$ $<<\tau_{\text {CLOSED }}$. For resistive reconnection, this requires reconnection in a cold zone feeding helicity into a hot zone. Here we propose an impedance model based on these ideas in a form that can be implemented in the Corsica-based helicity transport code. The most important feature of the model is the possibility that $\tau_{\mathrm{REC}}$ actually increases as the spheromak temperature increases, perhaps accounting for the "voltage sag" observed in some experiments, and a tendency toward a constant ratio of field to current, $\mathrm{B} \propto \mathrm{I}$, or $\mathrm{I}_{\text {TOR }}$ $\approx$ I. Program implications are discussed.
\end{abstract}

\title{
1. Fundamentals
}

High current amplification is an important goal in developing spheromaks as compact fusion reactors requiring 10's of MA of circulating current. It is also important that current be injected efficiently, requiring a power supply with impedance less than the spheromak impedance.

The conservation of helicity places strong conditions on achieving these goals. This can be seen by expressing helicity as an eigenfunction expansion of the field, $K=\Sigma_{i} \lambda_{i}^{-1} B_{i}{ }^{2}$, showing that most of the helicity lies in the axisymmetric mean field -- mainly the lowest mode $\lambda_{\mathrm{o}}-$ since other $\lambda_{\mathrm{i}}$ 's are larger and oscillate in sign [1], giving: 


$$
\mathrm{K}_{\mathrm{MEAN} \mathrm{FIELD}} \quad \approx\left(2 \mu_{\mathrm{o}} / \lambda_{\mathrm{o}}\right) \mathrm{E} \quad \approx\left(\mu_{\mathrm{o}} / \lambda_{\mathrm{o}}\right)^{2} \mathrm{I}_{\mathrm{TOR}}^{2}
$$

with mean field energy $\mathrm{E}$, mean field toroidal current $\mathrm{I}_{\mathrm{TOR}}$ and a constant geometric factor also reflecting the "mean field" $\lambda_{\mathrm{o}}$. Helicity on the open-line flux core of length $\mathrm{L}$ is the product of the bias flux $\psi$ carried forward by the flux core and flux due to the gun current [2]:

$$
\mathrm{K}_{\mathrm{OPEN}} \quad \approx \quad\left[\left(\mu_{\mathrm{o}} \mathrm{I} / 2 \pi \mathrm{a}\right) \mathrm{La}\right] \psi=\left(\mu_{\mathrm{o}} / \lambda_{\mathrm{o}}\right)^{2} \mathrm{I}^{2}
$$

where $\mathrm{a}$ is the flux core radius and in the second step we postulate that the flux core length stretches (like a "doughhook") in proportion to current when $I>I_{0}=\left(\lambda_{0} / \mu_{0}\right) / \psi$-- the case of interest -- giving $\mathrm{L} \approx \pi \mathrm{R}\left(\mathrm{U}_{\mathrm{o}}\right)$ with flux conserver radius $\mathrm{R}$. Both Eq. (1), and also Eq. (2) concerning $L \propto I$, are conjectural, though there is much experimental evidence supporting Eq. (1) and we suspect there is also for Eq. (2), which does explain NIMROD results.

It follows from Eqs. (1) and (2) that, in the absence of reconnection so that $\mathrm{K}=$ $\mathrm{K}_{\text {MEAN FIELD }}=\mathrm{K}_{\text {OPEN }}$, the mean field $\mathrm{B} \propto \mathrm{I}$, or $\mathrm{I}_{\text {TOR }} \approx \mathrm{I}$, giving little current amplification. It is this that explains the absence of current amplification in NIMROD simulations though there is flux amplification [3], with the bonus that -- because all lines are open -- one can also understand why the line length, properly averaged, is just the flux core length $\mathrm{L} \approx$ $\pi R\left(I / I_{0}\right)$ postulated above $[3,4]$.

More importantly, since without reconnection there is little current amplification, achieving high current amplification -- if this is possible -- must require reconnection in which helicity is transferred from open lines to closed lines, described by:

$$
\mathrm{dK}_{\mathrm{CLOSED}} / \mathrm{dt}=\mathrm{K}_{\mathrm{OPEN}} / \tau_{\mathrm{REC}}-\mathrm{K}_{\mathrm{CLOSED}} / \tau_{\mathrm{CLOSED}}
$$

with reconnection time $\tau_{\text {REC }}$ and ohmic dissipation time $\tau_{\text {CLOSED }}$ on closed field lines. Now identifying $\mathrm{K}_{\text {CLOSED }}$ with $\mathrm{K}_{\text {MEAN FIELD }}$ in Eq. (1) and taking $\mathrm{K}_{\mathrm{OPEN}}$ from Eq. (2), this gives the equation in the Abstract: 


$$
\mathrm{dI}_{\mathrm{TOR}}{ }^{2} / \mathrm{dt} \quad \approx \quad \mathrm{I}^{2} / \tau_{\mathrm{REC}} \quad-\quad \mathrm{I}_{\mathrm{TOR}}{ }^{2} / \tau_{\mathrm{CLOSED}}
$$

Then high current amplification requires:

$$
\tau_{\mathrm{REC}} \quad \ll \quad \tau_{\mathrm{CLOSED}}
$$

Thus we are led to the conclusions stated in the Abstract. Namely, achieving high current amplification requires reconnection at a rate that suppresses buildup of $\mathrm{K}_{\text {OPEN }}$ in favor of $\mathrm{K}_{\mathrm{CLOSED}}$, at a rate that is faster than the ohmic dissipation of $\mathrm{K}_{\mathrm{CLOSED}}$. Moreover, if reconnection is also ohmic in origin (tearing), then reconnection must be localized in a region that remains cold as the closed region heats up. For example, if reconnection were localized to the cold flux core (FC) as postulated in Ref. [3], in steady state Eq. (3) gives:

$$
\mathrm{K}_{\text {CLOSED }}=\left(\mathrm{T} / \mathrm{T}_{\mathrm{FC}}\right) \mathrm{K}_{\mathrm{OPEN}}
$$

using $\tau_{\text {CLOSED }} \propto T \checkmark T_{F C}$ for a parabolic profile with peak temperature $T$. Then $K_{\text {CLOSED }}$ representing the spheromak grows if $\mathrm{T}$ grows; and if $\mathrm{T} \propto \mathrm{B}^{2} \propto \mathrm{K}$ (fixed beta) there is no steady state, typical of optimistic predictions in SPHERE simulations in the past.

On time-average, these conclusions apply equally to continuous injection or to injection in "bursts," until reconnection occurs. For example, by Eq. (3), too rapid injection of spheromaks to create a larger one would fail -- either because later pulses would not enter the flux conserver or, if they did, they would mainly produce turbulence with no increase in helicity.

Continuous or pulsed, successful buildup to high current amplification must proceed at the reconnection rate.

\section{Impedance Model}

The results of Section 1 can be used to construct an equation for the gun voltage and impedance, giving:

$$
2 \psi\left(\mathrm{V}-\mathrm{V}_{\text {SHEATH }}\right)=\left\{\mathrm{dK}_{\mathrm{OPEN}} / \mathrm{dt}+\mathrm{K}_{\mathrm{OPEN}} / \tau_{\mathrm{REC}}\right\}+\mathrm{K}_{\mathrm{OPEN}} / \tau_{\mathrm{OPEN}}
$$

The last term on the right is ohmic loss in the flux core while for resistive reconnection 
$\mathrm{K}_{\mathrm{OPEN}} / \tau_{\mathrm{REC}}$ is the Enhanced Resistance model of Ref. [4], for which experimental evidence is reviewed in Section 3. The term $\mathrm{dK}_{\text {OPEN }} / \mathrm{dt}$ represents inductance in the flux core, including MHD distortions of field lines (the "doughhook" phenomenon).

To use Corsica for data analysis including our impedance model, as the boundary condition for the Corsica equilibrium we should use the terms in \{ \}'s in Eq. (7) to calculate the helicity input rate at the separatrix between open and closed flux calculated by Corsica. In the 2D Corsica, this boundary does not mark the true boundary between open and closed flux since in 3D open field lines from the flux core may penetrate the Corsica closed boundary, as they do in NIMROD, and the phenomenological hyper-resistivity coefficient $\Lambda$ in Corsica does not distinguish between these open line excursions and helicity transport by resistive reconnection described by our Enhanced Resistance term.

To account for both processes, the Corsica boundary condition should be:

$$
\int \mathrm{d} \mathbf{S} \cdot \Lambda(-\nabla \lambda)=2(\Delta \mathrm{V})_{\mathrm{CLOSED}} \psi=2 \psi\left\{\left(\mathrm{P}_{\Omega} / \mathrm{I}\right) \mathrm{KF}+\left(\mu_{\mathrm{o}} / \lambda_{\mathrm{o}}\right)\left(\mathrm{I} / \mathrm{t}_{\mathrm{o}}\right)(\mathrm{d} \mathrm{I} / \mathrm{dt})\right\}
$$

where the inductive term $\propto \mathrm{d} / \mathrm{dt}$ comes from $\mathrm{dK}_{\text {OPEN }} / \mathrm{dt}$ using Eq. (2), and the term $\propto \kappa$ is the Enhanced Resistance term given by:

$$
\begin{aligned}
& \mathrm{K}_{\mathrm{OPEN}} / \tau_{\mathrm{REC}}=2 \psi\left(\mathrm{V}-\mathrm{V}_{\mathrm{SHEATH}}\right)_{\mathrm{RESIS}}=2 \psi\left(\mathrm{P}_{\Omega} / \mathrm{I}\right) \mathrm{KF} \\
& \mathrm{F}=1 / 2\left\{1-\left(\mathrm{I}_{\mathrm{o}} / \mathrm{I}\right)^{2}\right\} \rightarrow 1 \text { for } \mathrm{I}>>\mathrm{I}_{\mathrm{o}} \\
& \mathrm{V}_{\text {SHEATH }}=\gamma \mathrm{T}_{\mathrm{FC}}
\end{aligned}
$$

where typically $\gamma=5-10 ; \mathrm{I}$ and $\mathrm{P}_{\Omega}$ are the current and ohmic power on open field lines calculated by Corsica; and $\mathrm{F}$ is a "free energy" factor.

The adjustable parameters in the boundary condition Eq. (8) are the coefficient $k$ and the gun current I. For constant current injection, the current must be continuously adjusted to equate the specified power supply current to that calculated by Corsica. For this case, the sheath voltage is not needed, but it would be if instead of constant I we determine I by a circuit equation in which $\mathrm{V}$ is the gun voltage. The flux core temperature $T_{F C}$ appearing in $V_{\text {SHEATH }}$ and also $P_{\Omega}$ is determined by ohmic heating and parallel heat loss, for 
which a formula could be provided [4], or $\mathrm{T}_{\mathrm{FC}}$ could be specified from experimental data. Finally, we could add Rechester-Rosenbluth heat transport and ion classical heat transport as we do in SPHERE, or simply use S-scaling, enhanced by the factor $g_{p}$ in SPHERE.

The impedance physics is contained in the resistance enhancement factor $\kappa$ discussed in Section 4. Our Enhanced Resistance model differs from the impedance model of Barnes et al [5] which requires mass flow, while resistive reconnection (tearing) is accomplished by resistive diffusion of the field with little mass transport at the point of tearing (perhaps explaining the difficulty of Barnes et al in reconciling mass throughput with impedance in CTX).

As in the Barnes et al model, we will assume that the threshold current $I_{0}$ is about constant, consistent with the observation that buildup of the spheromak tends to persist as long as I exceeds $I_{o}$ and it tends to cease at about the same current as that at which buildup commences. In our model, $I_{o}$ enters two physically-different ways, first in the inductive term (MHD), and secondly in the reconnection free energy factor $F$ (tearing).

\section{Experimental Evidence}

Like the model of Barnes et al [5], the Enhanced Resistance model says that the helicity input is maximum at $I \approx 2 I_{0}$, which appears to explain the typically higher impedance with $400 \mathrm{kA}$ from the Formation Bank compared to a drop in impedance during Sustainment at $200 \mathrm{kA}$ when the bias is set to optimize formation at high current but not sustainment at low current.

In Ref. [4] we pursued this point in some detail by applying the Enhanced Resistance model to SSPX data at fixed bias and a range of currents. Results are given in Figure 1 taken from Figure 13 of Ref. [4], where the highest "sustainment" points are splitbank shots. The model gave a good fit both to Formation Bank and Sustainment Bank data, with a classical resistance $R_{F C}=0.5 \mathrm{~m} \Omega$.

These results were obtained with a constant fitting parameter, $\kappa=4.86$, related in Ref. [4] to Rutherford island growth. A different insight into the meaning of $\kappa$ can be obtained from spectral expansions of the magnetic field in curl eigenstates [1]. For resistive reconnection, $\kappa$ represents tearing at a rate determined by the ohmic dissipation of fluctuations through the resistive term in Faraday's Law. In spectral notation, this term projected onto the ith eigenstate gives: 


$$
(\nabla \times \eta \mathbf{j})_{\mathrm{i}} \quad=\quad \Sigma_{\mathrm{j}} \lambda_{\mathrm{i}} \lambda_{\mathrm{j}} / \eta \mathbf{B}_{\mathrm{i}} \cdot \mathbf{B}_{\mathrm{j}} \quad \equiv \quad<\lambda_{\mathrm{FLUC}}^{2} \eta>
$$

That is, the enhanced resistance must be averaged over dissipation of modes that couple to the flux core but reach into the closed volume where temperatures are higher and changing in time. The depth of penetration is $1 / \lambda_{\text {FLUC }}$ where $\lambda_{\text {FLUC }}$ is that for the typical tearing mode, giving a temperature in the reconnection zone of order $\mathrm{T}_{\mathrm{REC}} \approx 2\left(\lambda_{\mathrm{o}} / \lambda_{\mathrm{FLUC}}\right) \mathrm{T}$ for a parabolic temperature profile. Then by the steps leading to Eq.(9):

$$
\kappa \quad \approx\left(\eta_{\mathrm{REC}} / \eta_{\mathrm{FC}}\right) \approx\left(\mathrm{T}_{\mathrm{FC}} / \mathrm{T}_{\mathrm{REC}}\right) \approx 2\left(\lambda_{\mathrm{FLUC}} / \lambda_{\mathrm{o}}\right)\left(\mathrm{T}_{\mathrm{FC}} / \mathrm{T}\right)
$$

As more is known about which tearing modes cause reconnection, we may be able to calculate $\kappa$ from first principles, along the lines of Ref. [1]. For now, we treat $\kappa$ as a fitting parameter.

With this interpretation, the fit with $\kappa=4.86$ corresponds to $\lambda_{\text {Fud }} / \lambda_{\mathrm{o}}=2.4$ by Eq. (13), early in time when $\mathrm{T} \approx \mathrm{T}_{\mathrm{FC}}$. This is perhaps a reasonable value, suggestive of the next highest energy level above the Taylor state.

The model did not fit the data for the Sustainment Bank near the threshold around I $\approx I_{o}$, where the measured impedance is quite erratic. This may or may not represent an opportunity to enhance $\kappa$, as Woodruff tried to do in Shot 7226 [6]. This shot may approximate repeated injection of finite "chunks" of helicity created if local tearing intermittently "necks off" a finite volume of plasma carrying helicity with it [6] -- giving as a signature the transport of mass with helicity as in the Barnes et al model. However, the impedance achieved thus far is still $\approx 2 \mathrm{~m} \Omega$, with voltage sagging during the shot, and the only observed measure of mass transport, the line density, was about constant.

\section{Sagging Voltage -- $\mathrm{B} \propto \mathrm{I}$}

The sag in voltage in Shot 7226 is correlated with an increase in the global decay time $\tau$, as shown in Figure 2, taken from Stallard's APS paper, November, 2001. This increase in $\tau$ presumably arises from an increase in spheromak temperature during buildup, not yet measured. To check this point, we have attempted to calculate temperatures in Shot 
7226 using the SPHERE code with an applied voltage $\triangle V=V-V_{\text {SHEATH }}$ that falls off in time:

$$
\Delta V=400 \text { volts }\left(1-t / t_{o}\right)
$$

where $t_{0}=3 \mathrm{~ms}$ (data from Barry Stallard's APS paper). The current was held constant at $200 \mathrm{kA}$ and the efficiency $\mathrm{f}$ of converting power into mean field energy was adjusted to give the observed magnetic field. The density was held constant at $\mathrm{n}=10^{20} \mathrm{~m}^{-3}$ and we did cases for $Z_{\text {eff }}=1$ and $Z_{\text {eff }}=2$. The case $Z_{\text {eff }}=2$ gives the correct peak field $B=0.2$ tesla for $\mathrm{fIV} \approx 20 \mathrm{MW}$ at $\mathrm{t}=0$. The implied efficiency $\mathrm{f}=0.25$ includes ohmic losses in the flux core and surroundings but not the sheath $(\approx 100$ volts) which was subtracted as in Stallard's paper.

Results are shown in Figures 3 and 4. Like Figure 2, the simulation shows an increasing decay time, accounted for by a rising temperature reaching a maximum $T=0.1$ $\mathrm{KeV}$ during buildup of the magnetic field. Here $\tau$ means $\tau_{\text {CLOSED }}$. This $\tau$ reached a maximum value of $1 \mathrm{~ms}$ at the peak field, compared to Stallard's global decay time $0.4 \mathrm{~ms}$ that includes ohmic losses in the flux core. Note also that the temperature doubles and $\tau$ doubles in the interval over which $\Delta \mathrm{V}$ falls by a factor of 2 , consistent with the experimental observation $\Delta V \tau \approx$ constant and our interpretation $\kappa \propto 1 / \tau \propto 1 / T$ as the explanation of "sagging voltage." In this shot, the density increased near the end of the shot. Both of these effects are included in Figure 5, where now Eq. (14) is replaced $\Delta V \propto$ $\kappa \propto 1 / \mathrm{T}$ and the density begins to rise at $\mathrm{t}=2 \mathrm{~ms}$.

If $\kappa \propto 1 / \mathrm{T}$, giving $1 / \tau_{\mathrm{REC}} \propto 1 / \mathrm{T}$, then, since also $1 / \tau_{\mathrm{CLOSED}} \propto 1 / \mathrm{T}$, by Eq. (3) steady state again gives $I_{\text {TOR }} \approx I(B \propto I)$, or using Eq. (13), $I_{\text {TOR }} \approx\left(\lambda_{\text {FLuC }} \lambda_{\mathrm{o}}\right)^{1 / 2}$ I. Thus once more we find limited current amplification, in this case determined by tearing mode geometry and the proximity of the flux core to the hot spheromak.

\section{Discussion -- Program Implications}

The impedance model presented here and experimental evidence supporting it raise profound questions for the program. On the one hand, the requirement that current injection proceed at an ohmic reconnection rate gives further credence to S-scaling and good heat 
confinement that was the original motivation for the SSPX program. On the other hand, it points up a not fully appreciated pitfall that can limit buildup to high magnetic fields for fundamental reasons. Low impedance and sagging gun voltage may simply be the consequences of consistently higher temperatures in SSPX.

Our first recommendation, given the evidence for the model to date, is that a systematic analysis be undertaken to confirm or discredit the model. This can begin with more analysis of $\Delta V \tau$, especially for shots in which $B$ plateaus as it did in Shot 7226.

Thomson measurements of $T$ during buildup could then be compared with $\tau$ from analysis to see if sagging voltage is really a symptom of rising temperature.

Our second recommendation is that the Corsica-based helicity transport code be modified as recommended here, in order to incorporate our model in more detailed data analysis. A comparison of $\Lambda$ obtained by the impedance model boundary condition Eq. (8) versus that obtained by fitting magnetic probe data would be instructive, as would a comparison of Corsica results with NIMROD simulations of injection at constant current.

Our third recommendation is to echo once again support for repeating the split-bank experiment as our best near-term route to higher fields and possibly higher temperatures, now using the Sustainment Bank for startup followed by the full Formation Bank.

Finally, we hope others will critically review our premises in Section 2 and, if they stand up, use them as a guide to improve performance in SSPX, or to motivate modifications.

Our main point, that transferring helicity stored on open lines to closed lines requires reconnection fast compared to ohmic dissipation in the spheromak, is not new but deserves much more thought if our interpretation of "sagging voltage" stands up. This means that, though the Taylor idea that the flux core would form a natural relaxation oscillator pumping helicity into the spheromak is basically correct, the proximity of this process to the hot spheromak places a fundamental limitation on current amplification, set by tearing mode wavelengths. Fast reconnection leading to high current amplification may not occur automatically. Maybe we must encourage it, either by some new invention, or by using traditional methods such as magnetic snipping or deflection, or gas or impurity puffs to interrupt the current as one extinguishes an arc.

\section{References}

[1] D. D. Hua, T. K. Fowler and E. C. Morse, "Magnetic Relaxation in Spheromaks Using Spectral Expansions in Cylindrical Geometry," Journal of Plas. Phys., in press. 
[2] T. R. Jarboe, Plas. Phys. Control. Fus. 36, 945 (1994).

[3] T. K. Fowler, "Consequences of Open Field Lines During Gun Injection into Spheromaks," 2nd Revision, November 2, 2000.

[4] T. K. Fowler, D. D. Hua and B. W. Stallard, "Simulations of SSPX

Sustainment -- Toward a Standard Model for Spheromaks," UCRL-ID-141998, January $12,2001$.

[5] C. W. Barnes, T. R. Jarboe, G. J. Marklin, S. O. Knox and I. Henins, Phys., Fluids B, 1871 (1990).

[6] Simon Woodruff, private communication.

This work was performed under the auspices of the U.S. Department of Energy by the University of California, Lawrence Livermore National Laboratory under contract No. W-7405-Eng-48. 
Fig. 1

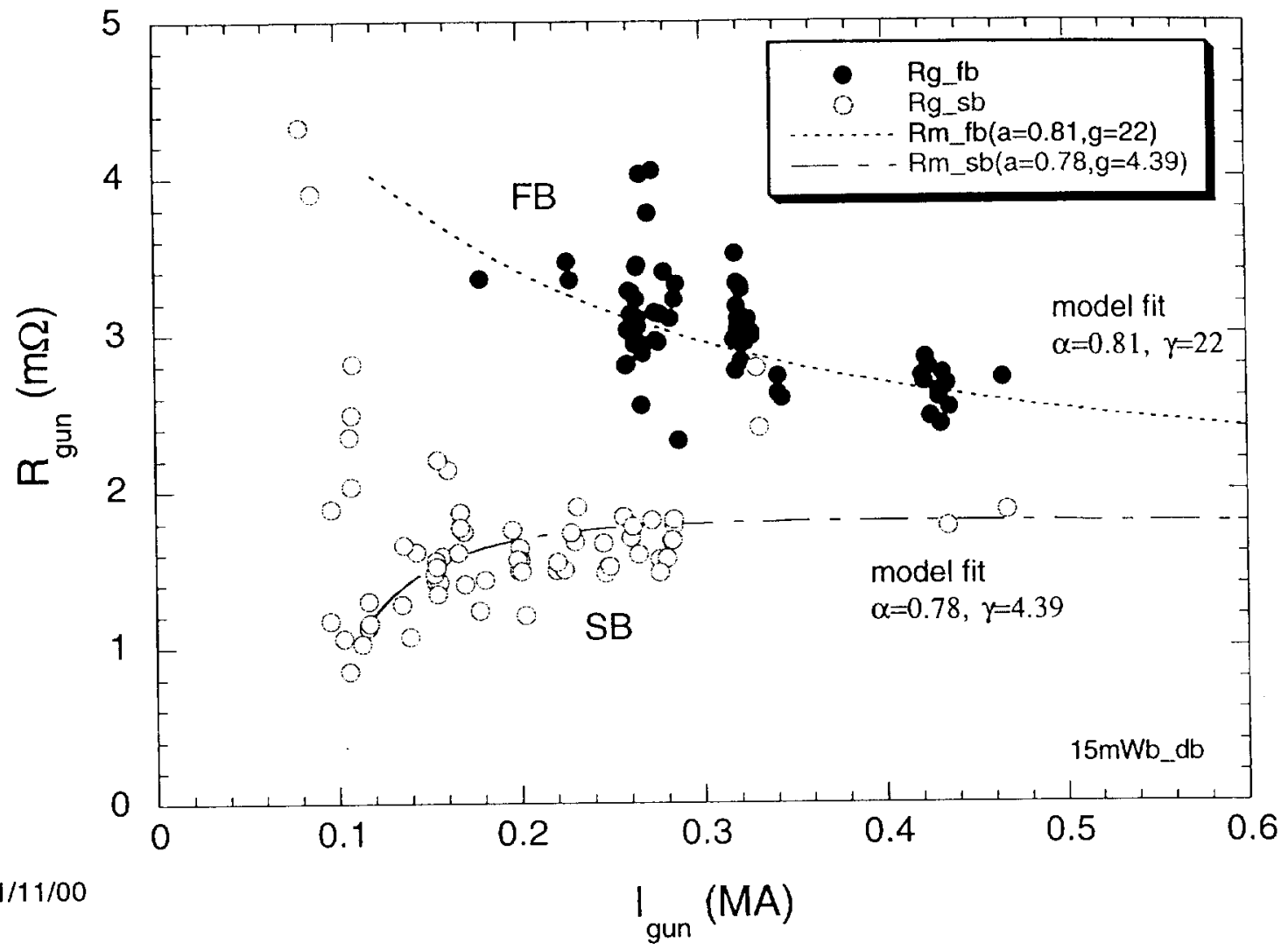


Fig. 2 Helicity Dissipation Decreases During Long Pulse

Long pulse buildup simulated by solution of the helicity balance equation $\left(\Delta \mathrm{V}_{\mathrm{sh}}=100 \mathrm{~V}\right)$ :

$$
\mathrm{dK} / \mathrm{dt}=2 \Psi_{\mathrm{g}}\left(\mathrm{V}_{\mathrm{g}}-\Delta \mathrm{V}_{\mathrm{sh}}\right)-\mathrm{K} / \tau_{\mathrm{K}}
$$

The Helicity dissipation rate inferred from the mhd equilibrium decreases during the pulse as gun voltage decreases $\left(\mathrm{T}_{\mathrm{e}}\right.$ data taken at $2 \mathrm{~ms}$ only).
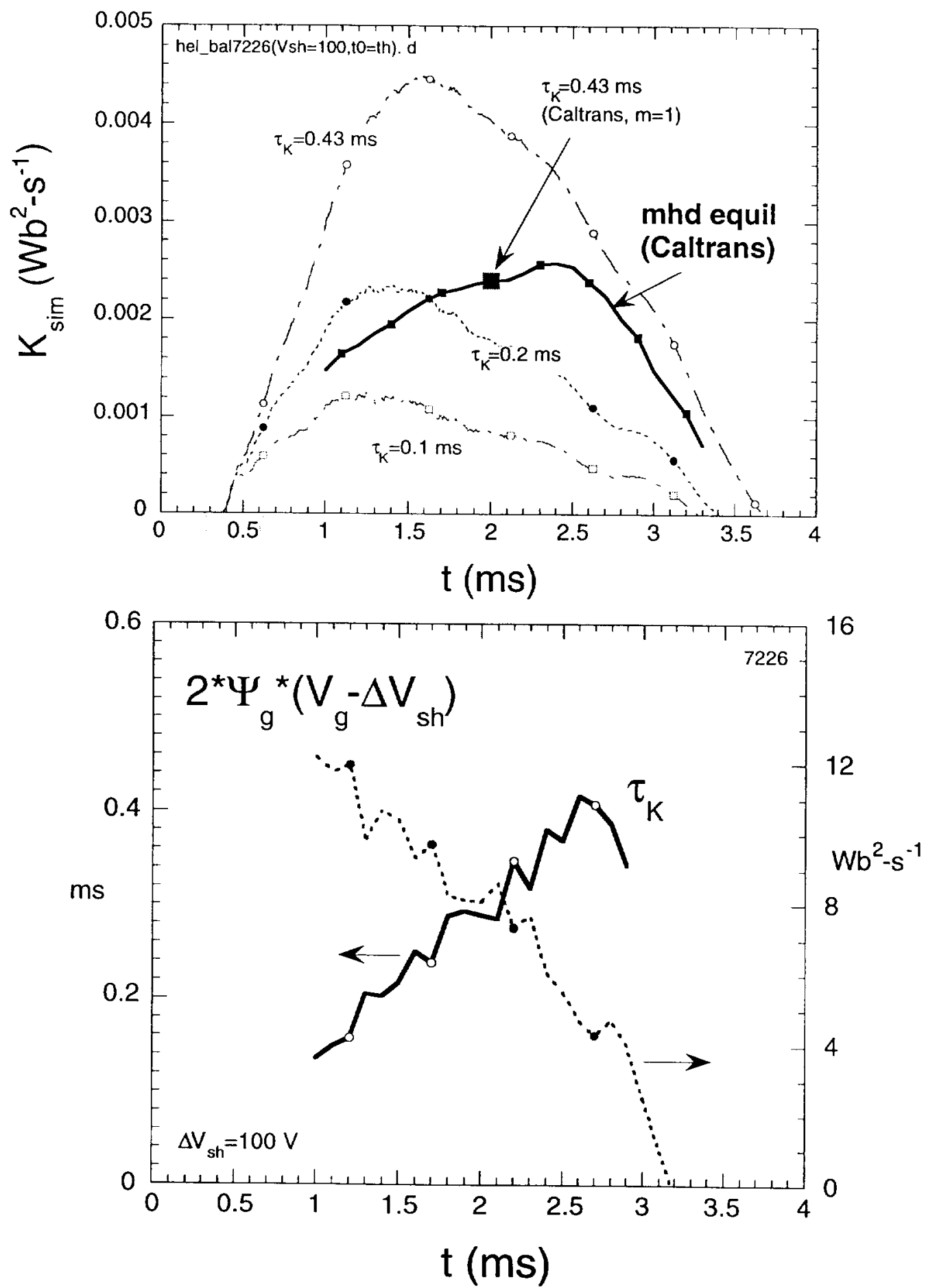
Fig. 3

SPHERE Simulation of Shot 7226

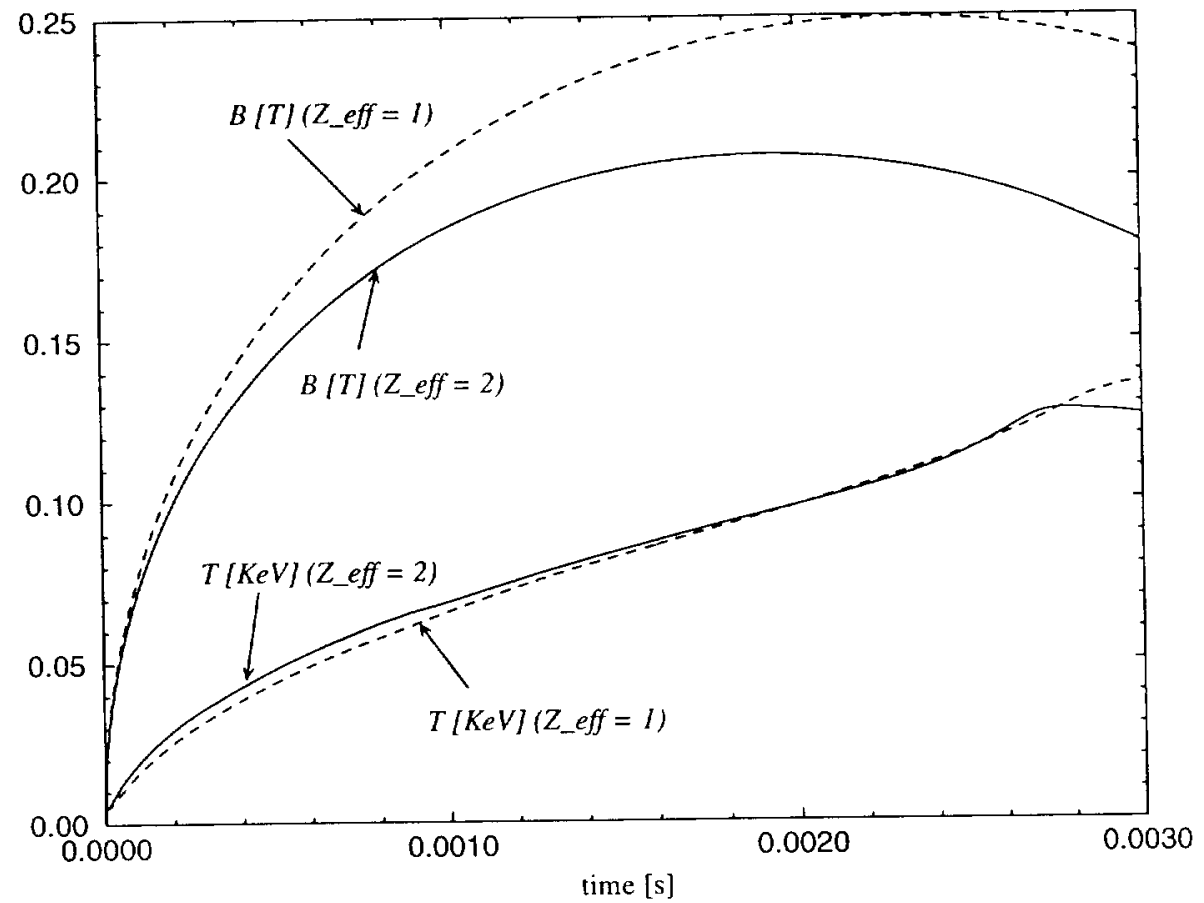

SPHERE Simulation of Shot 7226

Fig. 4

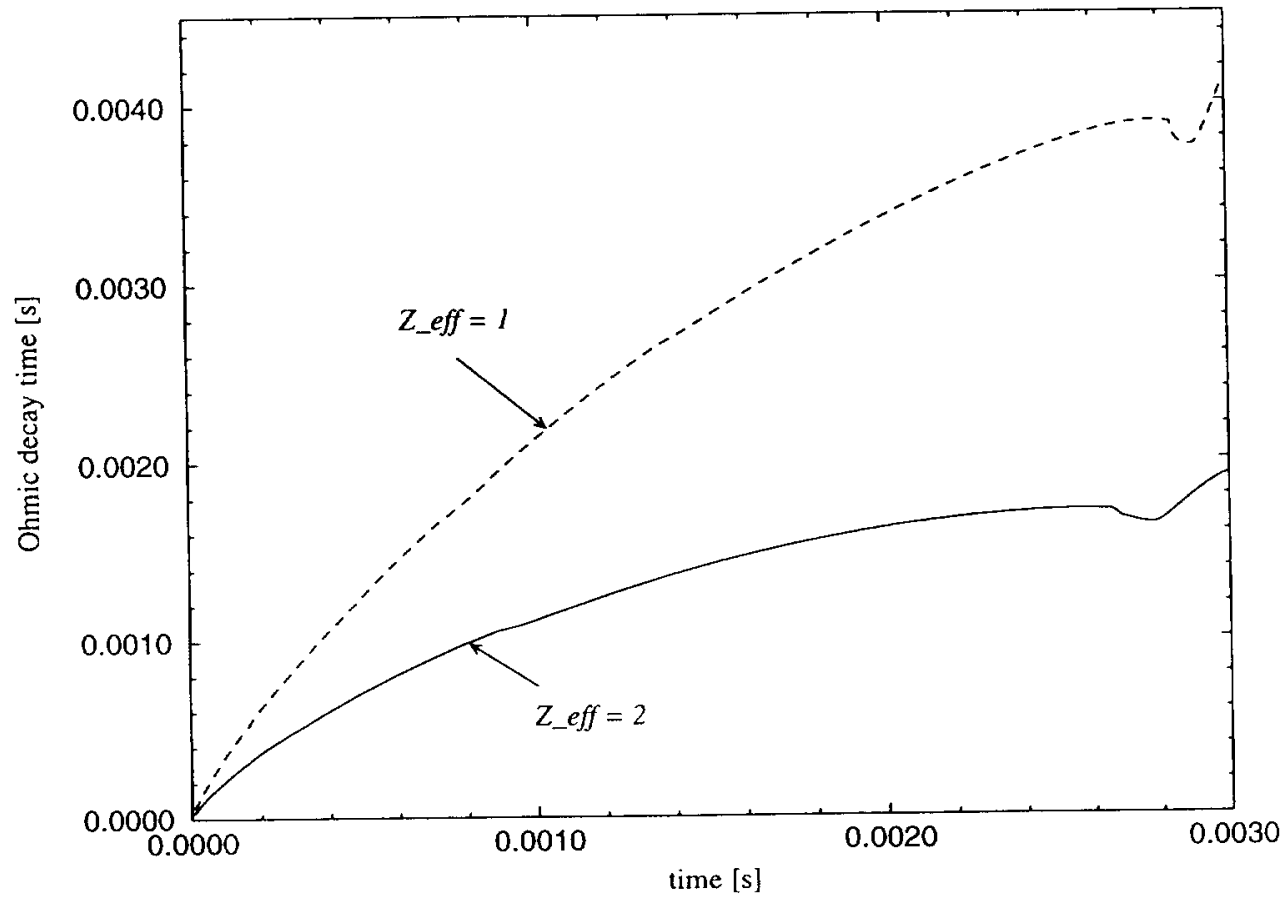


Fig. 5

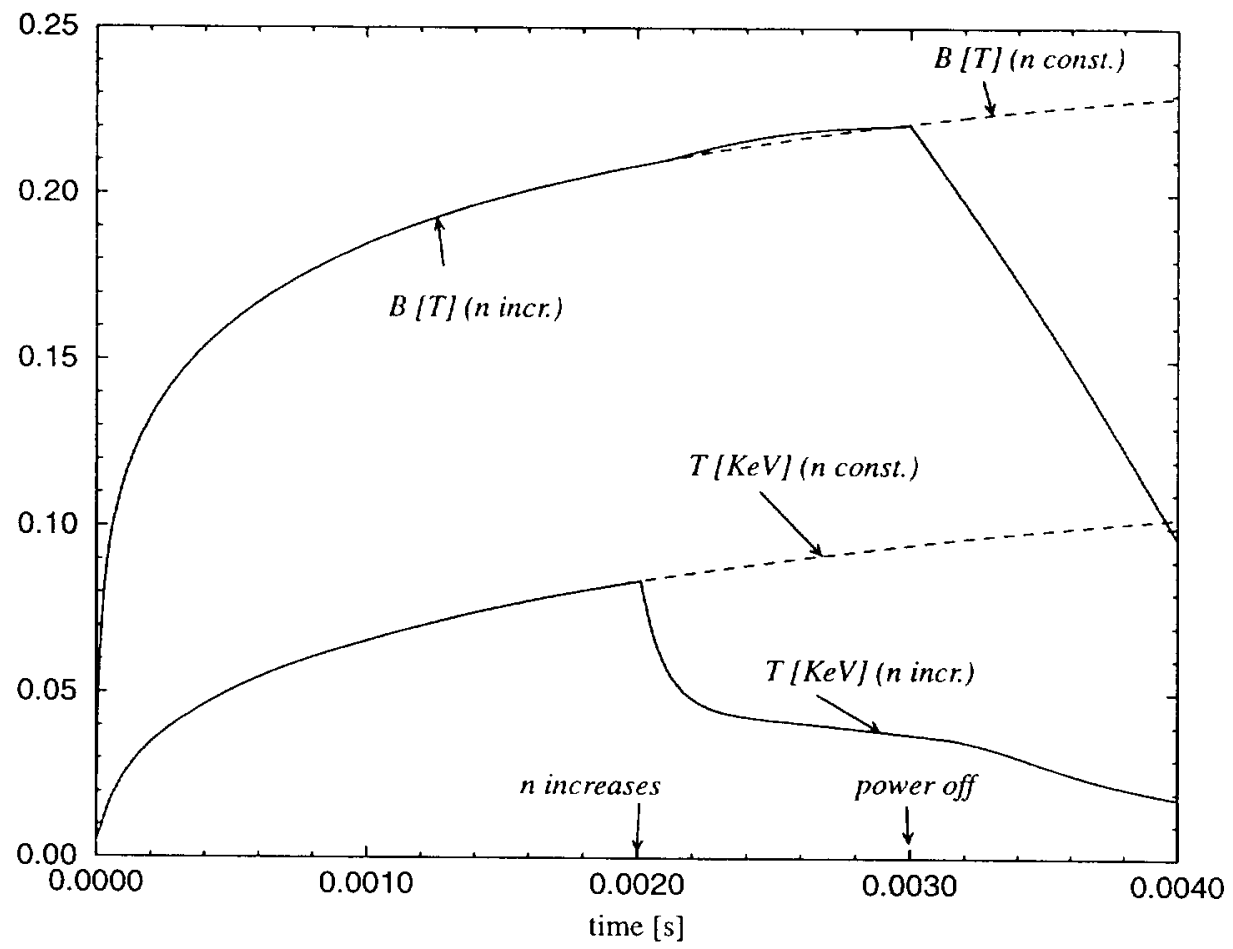

